\title{
Colpoperineocleisis de Labhardt: una alternativa segura y eficaz para el tratamiento del prolapso genital en pacientes sin actividad sexual
}

Javier Pizarro-Berdichevsky. ${ }^{1}$, Gonzalo Galleguillos L. ${ }^{1}$, Rodrigo Cuevas G. ${ }^{1}$, Marco Aramayo R. 1, Bernardita Blümel M. 2, Alejandro Pattillo G. 1, Silvana González L. a, Alejandro Majerson G. b, Jacobo Alvo J. b, Gonzalo Valdevenito A. 3, Mauricio Cuello F. 4 , Juan Andrés Ortiz C. 1

1 Unidad Uroginecología, Complejo Asistencial Dr. Sótero del Río (SSMSO), División de Obstetricia y Ginecología, Facultad de Medicina, Pontificia Universidad Católica de Chile. ${ }^{2}$ Unidad Uroginecología, (SSMSO). ${ }^{3}$ Unidad de Urología, (SSMSO). ${ }^{4}$ Unidad de Ginecología, División de Obstetricia y Ginecología, Facultad de Medicina, Pontificia Universidad Católica de Chile.

a Matrona, Unidad Uroginecología, Complejo Asistencial Dr. Sótero del Río (SSMSO).

b Alumno, Escuela de Medicina, Pontificia Universidad Católica de Chile.

\section{RESUMEN}

Introducción: El prolapso genital impacta negativamente la calidad de vida y autoimagen de la mujer. Rutinariamente indicamos la colpoperineocleisis de Labhardt a pacientes de edad avanzada y sin actividad sexual. Objetivo: Evaluar los resultados con esta técnica e identificar posibles factores de riesgo que predigan la falla. Métodos: Análisis retrospectivo de pacientes tratadas con la técnica entre los años 2008-2010. Se realizó análisis univariado y multivariado para identificar factores de riesgo asociados a la falla y se construyeron curvas libre de falla anatómica a fin de evaluar la durabilidad. Resultados: 68 pacientes cumplieron los criterios de inclusión. La edad promedio fue $71,9 \pm 5,7$ años; $95,6 \%$ fueron clasificadas en etapa III o IV según POP-Q. El tiempo operatorio fue de $54 \pm 20$ minutos. Seguimiento promedio de 21 meses; la tasa de recidiva anatómica fue de $14,7 \%$. La tasa de incontinencia de orina de esfuerzo (IOE) de novo fue de 5,9\%. El análisis univariado identificó como factor predictor de recidiva el flujo vaginal posoperatorio; en análisis multivariado de Cox, esa condición mantuvo significancia estadística $(\mathrm{OR} 6,3)$. Se identificó como factor de riesgo el no acatamiento de la técnica quirúrgica (OR 7,2). Conclusión: La colpoperineocleisis de Labhardt es una técnica segura, con baja tasa de complicaciones, una tasa de recurrencia aceptable y baja tasa de IOE de novo. Pilar del éxito es evitar la infección y respetar la técnica quirúrgica. Es necesario estudios comparativos con otras técnicas oclusivas, como también evaluar el rol de la infección como causa de falla de la técnica.

\section{PALABRAS CLAVE: Prolapso de órganos pélvicos, cirugía uroginecologica, calidad de vida,} ancianidad lábil

\section{SUMMARY}

Introduction: Genital prolapse affects up to $50 \%$ of multiparous women, impacting their quality of life and selfimage. At our Institution Labhardt's colpoperineocleisis is usually proposed to older patients, without sexual activity, regardless the type or grade of prolapse. Objective: To evaluate the results with this technique and identify potential risk factors predicting the failure. Methods: Analysis of a retrospective cohort of patients 
treated with this technique between 2008 and 2010. Univariate and multivariate analysis were carried out to identify risk factors and failure-free curves were built to estimate durability. Results: 68 patients met the inclusion criteria. Average age was $71.9 \pm 5.7$ years; $95.6 \%$ of patients were classified as stage III or IV based on POP-Q system. Operative time was $54 \pm 20 \mathrm{~min}$. At 21 months of follow-up, recurrence rate was $14.7 \%$. De novo stress urine incontinence (SUI) was diagnosed in $5.9 \%$ of patients. In univariate analysis, post operative vaginal discharge was identified as predicting factor for defect recurrence. In Cox analysis, this condition remained significant (OR 6.3), In addition, failure to observe the surgical technique was also identified as risk factor for defect recurrence (OR 7.2). Conclusion: Labhardt 's colpoperineocleisis is a safe technique, with low complication rate, acceptable recurrence rate, and low de novo SUI rate. Mainstay for success is to avoid infection and observe the surgical technique. Comparative studies with other occlusive techniques might be carried out, as well as exploring the role of infection as predisposing condition for failure.

\section{Palabras Clave: Pelvic organ prolapse, urogynecological surgery, quality of life, frail elderly}

\section{INTRODUCCIÓN}

El prolapso de los órganos pelvianos (POP) corresponde al descenso de éstos, desde su ubicación original, a través de la vagina. Se estima que esta condición afecta hasta el $50 \%$ de las multíparas en distinto grado de severidad (1). Dependiendo de dicha severidad, puede deteriorar la calidad de vida de las pacientes incluyendo distintas esferas de ella, dentro de las cuales destacan los efectos sobre la autoimagen y la dinámica de interacción social $(2,3)$.

En Estados Unidos de Norteamérica se realizan aproximadamente 225.000 cirugías por POP al año (4). Se estima que el riesgo de una mujer de ser operada por esta causa aumenta con la edad. Así el riesgo de una paciente a los 80 años alcanza el $11 \%$ (5). En este sentido, para el año 2030 se estima que un quinto de la población de dicho país tendrá más de 65 años y por ende el número de mujeres mayores de 84 años superará los 6 millones (6). Ello avala el carácter epidémico que esta enfermedad tendrá en el futuro. En Chile no existen datos sobre la prevalencia del POP o del número de intervenciones originadas en esta causa. De acuerdo a las estadísticas locales más recientes de nuestro centro, la unidad de uroginecología realiza alrededor de 340 cirugías por prolapso y/o incontinencia urinaria al año (datos no publicados sobre la estadística registrada para el período correspondiente al año 2011), que representa el $44 \%$ del total de cirugías electivas ginecológicas realizadas.

Diversas técnicas han sido descritas para la reparación del POP. Dentro de ellas, las más utilizadas son las técnicas reconstructivas las cuales utilizan tejidos propios de la pacientes, estructuras de fijación autólogas o incorporan material protésico con la finalidad de reparar el defecto, preservar o restablecer tanto la anatomía como la funcionalidad del piso pelviano (7). Estas técnicas son de elección en pacientes quienes se encuentran sexualmente activas y que desean mantener dicha condición. Estas técnicas, aunque ofrecen esas ventajas, exponen potencialmente a las pacientes a un riesgo de recurrencia mayor.

En pacientes sexualmente inactivas, donde preservar la función sexual no tiene tal relevancia, el objetivo terapéutico prioriza el lograr la mayor eficacia y durabilidad en la reparación del defecto (8). Para este grupo de pacientes, donde se concentran habitualmente las de edad más avanzada y de mayor riesgo quirúrgico, es donde las técnicas oclusivas ofrecerían un mayor beneficio (9). Dentro de dichas técnicas se encuentran la colpocleisis de Le Fort, la más comúnmente utilizada, la colpocleisis de Conill, la colporrafia de Doderlein, y diversas modificaciones de las mismas (10). El principio quirúrgico que las rige es la oclusión total del tubo vaginal a través de la unión de tejido tegumentario y mucoso que constituye el cilindro vaginal, con escaso refuerzo muscular. Su mayor desventaja está en la alta tasa de incontinencia urinaria (IO) posoperatoria, debido al cambio en la posición y ángulo del cuello vesical (11).

Con la finalidad de disminuir la 10 postoperatoria se han introducido modificaciones a la técni$\mathrm{ca}$, donde se incluye la realización de colpocleisis parciales, preservando indemne el tercio distal de la pared anterior vaginal. La introducción de tal modificación, sumado a que esta técnica se realiza habitualmente en mujeres de edad avanzada donde los tejidos se encuentran con frecuencia atróficos, hace que la tasa de recurrencia de POP sea muy variable, llegando hasta un $30 \%$ dependiendo de la serie (11-14).

Dado que los resultados históricos en nuestra 
población eran similares a los descritos con otras técnicas por otros grupos, nuestro equipo decidió adoptar una antigua técnica oclusiva (o de colpoperineoclisis) en pacientes con POP sin actividad sexual. La técnica a la cual hacemos mención es la colpoperineoclisis de Labhardt, descrita por Alfred Labhardt en 1923. En esta técnica la oclusión vaginal conlleva la fusión de las paredes laterales y el introito vaginal (15). Dentro de las ventajas descritas con esta técnica se incluirían: la baja tasa de recidiva e IO postoperatoria, una corta curva de aprendizaje de la técnica, su bajo costo y tiempo operatorio menor con escasas pérdidas sanguíneas (16-18).

El objetivo del presente estudio fue evaluar nuestros resultados con esta técnica, aplicada a pacientes de edad avanzada, con POP, sin actividad sexual y su eficacia a corto y mediano plazo, en términos de durabilidad de la corrección del defecto y de la incidencia de incontinencia de orina postoperatoria.

\section{PACIENTES Y MÉTODO}

Para realizar el presente estudio, seleccionamos una cohorte retrospectiva de pacientes con $\mathrm{POP}$, quienes siendo de edad avanzada y sexualmente inactivas hubiesen sido sometidas a colpoperineoclisis de Labhardt. El protocolo de este estudio fue revisado y aprobado por el comité de ética científico del Complejo Asistencial Hospital Dr. Sótero del Río.

Criterios de inclusión: pacientes sometidas a la técnica de colpoperineoclisis de Labhardt, haber sido operada posterior al 31 de diciembre de 2007 (periodo en el cual se implementó la base de registro de datos de nuestra unidad) y tener un mínimo de 6 meses de seguimiento.

Criterios de exclusión: haber sido sometida a otra técnica de colpocleisis, el no contar con información suficiente en el protocolo quirúrgico que especificara la técnica usada, cirugía realizada antes de enero de 2008 y seguimiento menor a 6 meses. No constituyó criterio de exclusión el que la cirugía hubiese sido efectuada en parte por un médico en curva de aprendizaje ya que en todo procedimiento se contó con la presencia de un cirujano entrenado a fin de garantizar la correcta ejecución de la técnica.

Cabe señalar que la unidad de uroginecología de nuestro Centro cuenta con una base datos en línea, estandarizada, donde a cada paciente se le asigna un número único o folio y donde se registran los datos de interés clínico y el seguimiento. Esto permite mantener la confidencialidad de la paciente durante el proceso de análisis de los datos. Dentro de las variables incluidas en el registro y que fueron selecccionadas para el análisis del estudio se incluyeron: la edad de la paciente, las comorbilidades (diabetes mellitus, hipertensión arterial, dislipidemia), el estado de menopausia, el uso de terapia de reemplazo hormonal, el hábito tabáquico, la paridad, el antecedente de parto instrumental, el peso máximo del recién nacido, el uso de paños o toallas, la nocturia (2 o más episodios de micción durante la noche), la historia de urgencia y/o urgeincontinencia, el antecedente de histerectomía vaginal o abdominal sin importar la causa de ella, el antecedente de cirugía de prolapso y/o incontinencia.

En relación al examen físico se incluyeron en el análisis las siguientes variables: índice de masa corporal (IMC), el puntaje de POP-Q (19), la presencia y tipo de prolapso multicompartimento.

Las variables quirúrgicas de interés fueron: técnica quirúrgica ajustada a protocolo, tiempo operatorio, pérdida sanguínea estimada, cirugía concomitante (histerectomía), complicaciones intraoperatorias y posoperatorias inmediatas (esto es hasta el momento del alta), primer cirujano, número de días en hospital posterior a la cirugía.

En el relación al seguimiento se incluyeron las siguientes variables: meses de seguimiento total, ocurrencia e intervalo a la recidiva (definida como prolapso que sobrepase introito y clasificada como asintomática, sintomática o que requiere resolución quirúrgica) y las complicaciones tardías (habitualmente posterior al alta e incluyendo la incontinencia posoperatoria). Dentro del seguimiento se incluyó un indicador de satisfacción del paciente poscirugía (se preguntó “¿está satisfecha con la cirugía que se le realizó?") y de cambio en la calidad de vida (se preguntó "¿se siente mejor, peor o igual que antes de operarse?").

Debido a que al momento del estudio no existía un registro adecuado de algunas variables, no fueron analizadas: la historia de constipación, el síndrome de intestino irritable, los episodios de infección urinaria en el último año, el uso de estrógenos tópicos, la episiotomía, la presencia de incontinencia fecal, la historia familiar de prolapso genital y/o incontinencia urinaria, la actividad física pesada, la tos crónica o el antecedente de enfermedad pulmonar obstructiva crónica.

El análisis se realizó dependiendo del tipo de variable estudiada. Para variables continuas e intervalares se utilizó el promedio \pm desviación estándar $( \pm D E)$, para las variables ordinales se calculó la mediana y los rangos intercuartiles (25 y 75 ) y para las nominales se calculó el porcentaje e intervalo de confianza al 95\%. La significancia estadística comparativa entre subgrupos, en análisis univariado, se evaluó según corresponde con el test de 
student o Mann-Whitney U (variables continuas, ordinales e intervalares) y con test de Chi cuadrado o Fisher Exact (variables nominales). Finalmente se construyeron curvas de sobrevida libre de recurrencia (según análisis de Kaplan-Meier) a fin de evaluar la durabilidad de esta técnica quirúrgica según variables como: edad, estado hormonal, cirujano (miembros staff de la unidad v/s médicos residentes; curva de aprendizaje), etc. La significancia estadística se estableció en $\mathrm{p}<0,05$. En caso de haber más de 10 recidivas se planificó realizar análisis multivariado con regresión de Cox. Dicho análisis incluiría además de la edad al momento de la cirugía, paridad, antecedente de parto instrumental, antecedente de histerectomía (independiente de la vía), antecedente de cirugía de prolapso, grado de prolapso, presencia de prolapso multicompartimental, cirugía realizada en curva de aprendizaje, cirujano entrenado versus bajo supervisión y cualquier variable con valor $p<0,08$ al momento del análisis univariado en las curvas de sobrevida. Se consideraron en todos los casos un nivel de significación del $95 \%$.

Técnica quirúrgica. Demarcación romboidal de mucosa vaginal posterior con electrobisturí cuyo vértice proximal se inicia a $1 \mathrm{~cm}$ bajo labio posterior del cérvix o cúpula hasta introito y $2 \mathrm{~cm}$ sobre el ano en la piel perineal (Figura 1A). Demarcación de mucosa vaginal lateral bilateral con electrobisturí desde unión de los dos tercios superiores de la demarcación de mucosa posterior hasta unión mucocutánea, $2 \mathrm{~cm}$ bajo meato uretral, en la parte interna del la- bio menor. Resección mucosa vaginal posterior y lateral demarcada exponiendo tejido submucoso y muscular en forma de estrella u hoja de Canadá (Figura 1B). Cierre de mucosa vaginal en el vértice más próximo al cérvix o cúpula con poliglactina 910 \#3-0 hasta punto donde se inicia resección lateral. Plicatura alta en línea media de porción puborrectal del elevador del ano y del músculo bulboesponjoso con polidioxanona \# 0 ó \#1 en 3 a 4 puntos dobles separados (Figura 1C). Plicatura superficial en línea media cubriendo puntos de polidioxanona con poliglactina 910 \#1 en 3 a 4 puntos separados Se completa cierre de mucosa vaginal y piel perineal con poliglactina 910 \#3-0 (Figura 2A). Imagen del periné posterior a 30 meses de seguimiento (Figura 2B).

\section{RESULTADOS}

En el período comprendido entre enero de 2008 y julio de 2010 se diagnosticaron 77 pacientes con $\mathrm{POP}$, sin deseos de actividad sexual y las cuales fueron sometidas a alguna técnica oclusiva para reparar el defecto. La indicación quirúrgica se basó en el diagnóstico de un prolapso genital sintomático, en etapa II o superior y en la cual la paciente manifestó su intención de no tener actividad sexual vaginal en el futuro. El antecedente de histerectomía no fue relevante al momento de decidir el tratamiento propuesto. Como requisito todas las pacientes contaron con citología cervical (negativa y vigente) y ultrasonografía transvaginal normal.

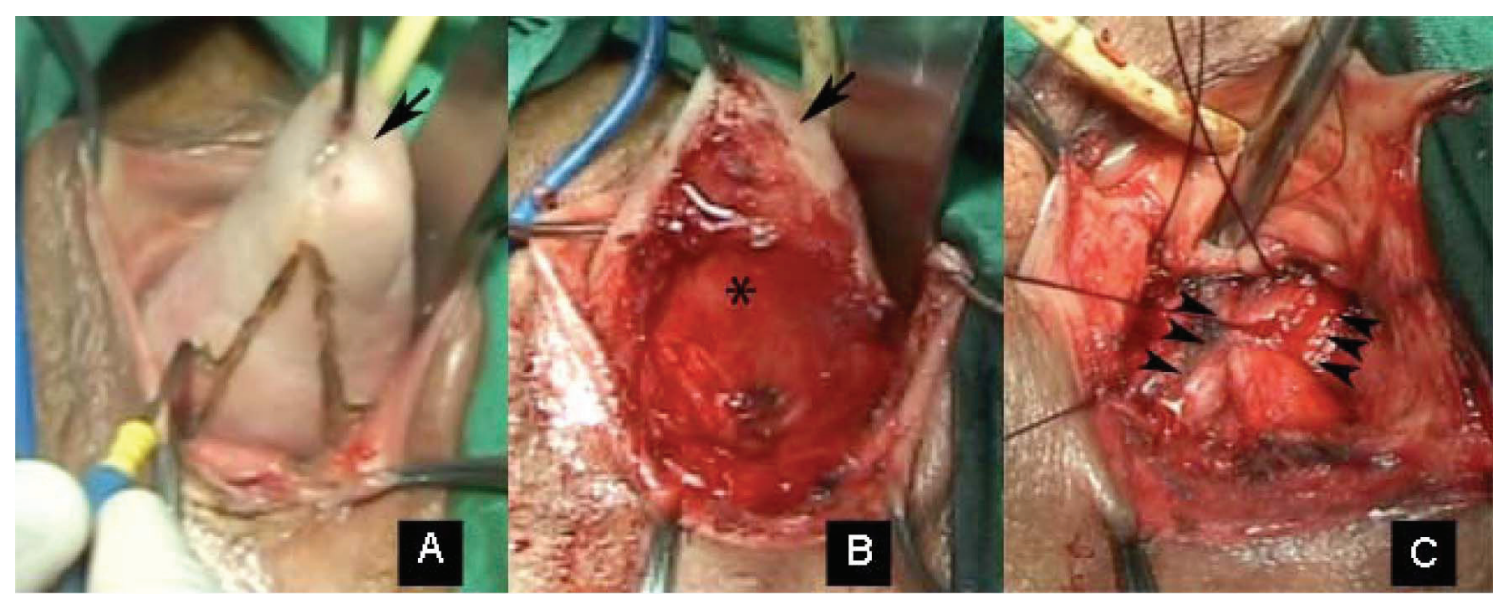

Figura 1. A) Demarcación romboidal de mucosa vaginal posterior con electrobisturí (flecha muestra cuello uterino traccionado con pinza Pozzi). B) Tejido submucoso y muscular expuesto pos resección de mucosa vaginal (asterisco muestra lecho cruento). C) Plicatura alta en línea media de porción puborrectal del elevador del ano (puntas de flecha muestran zona de plicatura). 


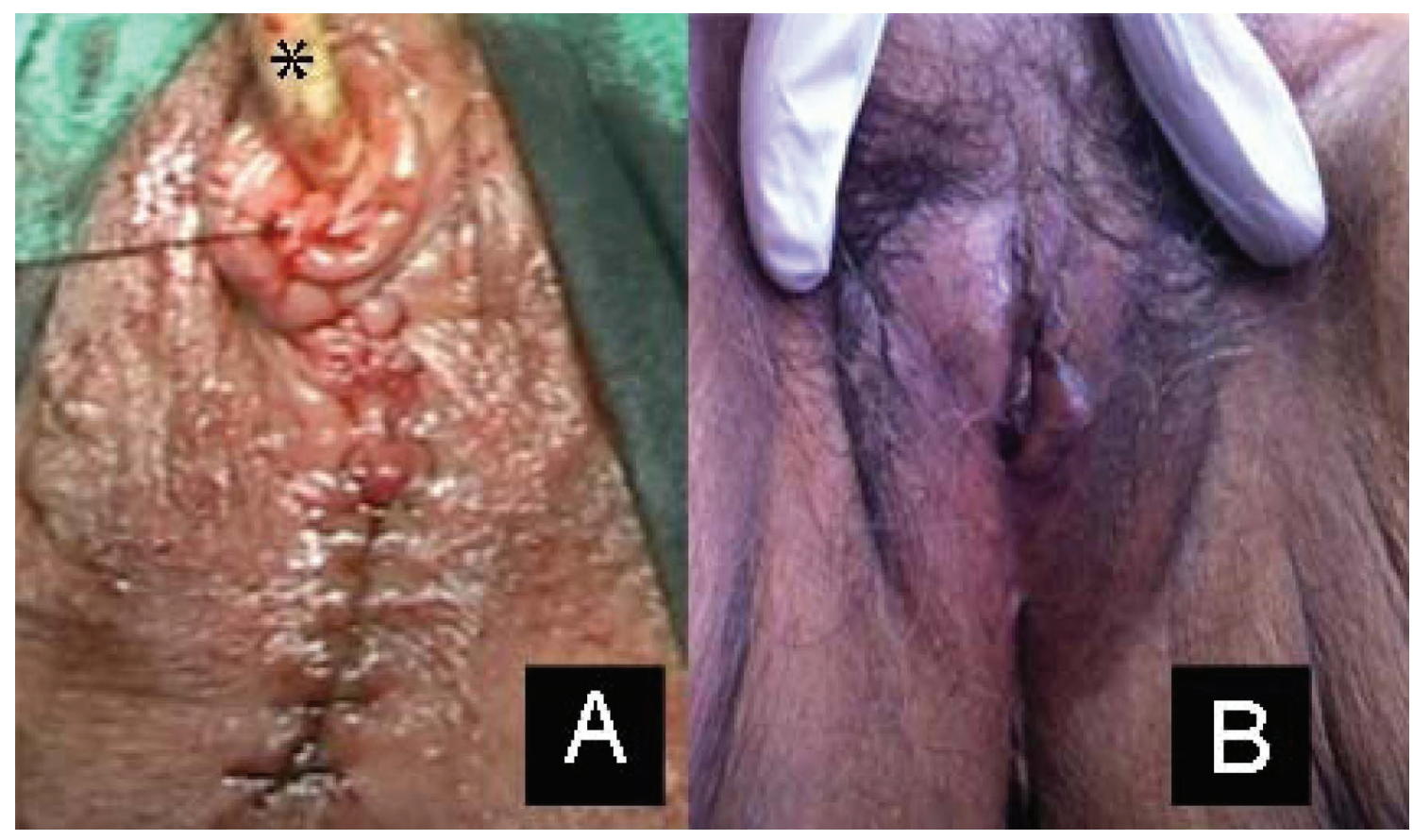

Figura 2. A) Aspecto externo al concluir cirugía (asterisco muestra sonda Foley in situ). B) Imagen del periné posterior a colpoperineocleisis de Labhardt a 30 meses de seguimiento.

De las 77 colpocleisis antes mencionadas, 71 fueron realizadas según la técnica de Labhardt y 6 correspondieron a la colpocleisis parcial de Le Fort. Fueron excluidas por seguimiento incompleto, 3 pacientes ( 1 de ellas falleció por una causa no relacionada a la cirugía y el resto se encontraba en buenas condiciones y sin recidiva al momento del ultimo control con promedio de seguimiento de 3 meses). Por tanto la serie incluida en el análisis fue de 68 pacientes.

Las características demográficas de las pacientes se describen en la Tabla I. En la evaluación preoperatoria el $95,6 \%$ de las pacientes se encontraban en etapa III o mayor de POP-Q. Para esta serie de pacientes, el punto de mayor prolapso fue apical, anterior y posterior en $33,3 \%, 62,1 \%$ y $4,5 \%$, respectivamente. En un $95,6 \%$ de los casos se evidenció un prolapso multicompartimental. De ellas, en 49 casos el defecto fue anterior, apical y posterior, en 9 casos anterior y apical y en 6 casos anterior y posterior. No se evidenció casos en que coexistiera un defecto posterior y apical. EI POP-Q promedio preoperatorio se describe en la Tabla II.

El análisis de variables intraoperatorias se resume en la Tabla III. Hubo una complicación intraoperatoria (sospecha de sutura transfixiante a la mucosa rectal). Se identificó 10 pacientes en las cuales no se siguió estrictamente el protocolo establecido de la cirugía. Dichas pacientes no se excluyeron del análisis por tratarse del reemplazo de polidioxanona 2-0 ó poliglactina 910 en vez de polidioxanona \#0 ó \#1 para la plicatura de los elevadores del ano. El tiempo promedio de permanencia en el hospital poscirugía fue 2,5 \pm 2,6 días (rango: 1-20 días). Se destaca el caso de una paciente con hospitalización prolongada (20 días), no por complicación de la cirugía sino por reinicio de tratamiento anticoagulante oral.

El seguimiento promedio fue $21,7 \pm 8,4$ meses (rango: 7-42 días). Diez pacientes presentaron recidiva anatómica (14,7\%), siendo todas sintomáticas. La mediana de seguimiento a la recurrencia fue 2 meses (rango: 0-33 meses). En 7 de estas pacientes se utilizaron las suturas adecuadas $(10,3 \%$ del total). En nueve de las recidivas se requirió de cirugía $(13,2 \%)$, realizándose en 8 de ellas nuevamente una colpoperineocleisis de Labhardt. Sólo en una paciente la corrección se realizó mediante la colpocleisis de Le Fort. Cuatro pacientes presentaron IOE de novo $(5,9 \%)$ durante el seguimiento.

Once pacientes presentaron flujo vaginal en el primer mes posoperatorio. En todas ellas se indicó tratamiento antibiótico, aunque sin confirmación microbiológica de infección. De éste grupo, 4 casos presentaron recidiva anatómica, correspondiendo a una tasa del $36,6 \%$ de falla.

En análisis univariado, de los potenciales factores 
Tabla I

\section{CARACTERÍSTICAS DEMOGRÁFICAS DE LAS PACIENTES SOMETIDAS A COLPOPERINEOCLEISIS DE LABHARDT}

\begin{tabular}{lc}
\hline Pacientes & $\mathrm{n}=68$ \\
\hline Edad & $71,9 \pm 5,7$ (R: $58-84$ años) \\
$\begin{array}{l}\text { Paridad (Hijos) } \\
\text { Mediana (IQ 25-75) }\end{array}$ & $4(2-6)$ \\
$\begin{array}{l}\text { Parto instrumental } \\
\mathrm{n}(\%)\end{array}$ & $14(20,6)$ \\
$\begin{array}{l}\text { Peso recién nacido } \\
\text { de >peso }\end{array}$ & $3694 \pm 680 \mathrm{~g}(\mathrm{R}: 2000-5500 \mathrm{~g})$ \\
$\begin{array}{l}\text { Antecedente de } \\
\text { histerectomía } \mathrm{n}(\%)\end{array}$ & $10(14,7)$ \\
Edad de menopausia & $46,5 \pm 5,9$ años \\
$\begin{array}{l}\text { Antecedente cirugía } \\
\text { de prolapso } \mathrm{n}(\%)\end{array}$ & $6(8,8)$ \\
IMC & $27 \pm 5,1(\mathrm{R}: 19-44 \mathrm{Kg} / \mathrm{m} 2)$ \\
\hline
\end{tabular}

Tabla II

POP-Q PROMEDIO PREOPERATORIO DE PACIENTES SOMETIDAS A COLPOPERINEOCLEISIS DE LABHARDT

\begin{tabular}{lr}
\hline $\mathrm{Aa}$ & $1,5 \pm 1,7$ \\
$\mathrm{Ba}$ & $3,6 \pm 2,2$ \\
$\mathrm{C}$ & $1,5 \pm 4,3$ \\
$\mathrm{gH}$ & $5,3 \pm 1,3$ \\
$\mathrm{pB}$ & $2,9 \pm 0,7$ \\
$\mathrm{TVL}$ & $8 \pm 1,2$ \\
$\mathrm{Ap}$ & $-0,6 \pm 2$ \\
$\mathrm{Bp}$ & $0 \pm 2,9$ \\
$\mathrm{D}$ & $-3,2 \pm 2,4$ \\
\hline
\end{tabular}

Datos presentados como promedio \pm DE en cm en relación al himen.

Tabla III

\section{VARIABLES INTRAOPERATORIAS DE PACIENTES SOMETIDAS A COLPOPERINEOCLEISIS DE LABHARDT}

\begin{tabular}{lc}
\hline Tiempo operatorio & $54 \pm 20(\mathrm{R}: 25-120 \mathrm{~min})$ \\
Pérdida sanguínea & $69 \pm 68(\mathrm{R}: 10-500 \mathrm{ml})$ \\
estimada & $8(11,8)$ \\
$\begin{array}{l}\text { Cirugía concomitante } \\
\mathrm{n}(\%)\end{array}$ & $50(73,5)$ \\
$\begin{array}{l}\text { Cirugía realizada por } \\
\text { cirujano staff } \mathrm{n}(\%)\end{array}$ & \\
\hline
\end{tabular}

de riesgo asociados a recidiva, sólo fue significativo la presencia de flujo genital requirente de tratamiento antibiótico $(p=0,049)$. El detalle del análisis de factores de riesgo se resume en la Tabla IV.

Al construir la curvas de sobrevida libre de recurrencia, se observa que a los 30 meses de seguimiento la falla observada llega a un $20 \%$ (Figura 3). Cuando se compara las curvas entre pacientes operadas ajustándose a la técnica vs aquellas en que se modificó mínimamente, esto es eligiendo suturas diferentes, observamos que la recurrencia fue de $30 \%$ a los 3 meses en el grupo en el cual no se siguió el protocolo, vs $13 \%$ a los 25 meses en el grupo donde la técnica quirúrgica fue adecuada. Pese a ello, la diferencia no fue significativa $(p=$ $0,057)$. En cuanto a la presencia de flujo genital, la recurrencia llega a un $36 \%$ a los 30 meses, valor más elevado, aunque no significativo $(p=0,088)$, comparado al $12 \%$ observado en el mismo periodo de seguimiento, en pacientes sin flujo. Finalmente, analizamos la sobrevida libre de recurrencia, a 30 meses, comparando cirugías realizadas por un médico entrenado vs uno supervisado en curva de aprendizaje. La tasa de recurrencia fue similar en ambos grupos (16\% vs $17 \%$ ). Tampoco encontramos diferencias significativas al analizar las cirugías realizadas dentro de la curva de aprendizaje y las realizadas posteriormente.

En el análisis de sobrevida multivariado de COX se incluyó la edad al momento de la cirugía, paridad, antecedente de parto instrumental, antecedente de histerectomía, antecedente de cirugía de prolapso, grado de prolapso, presencia de prolapso multicompartimental, cirugía realizada en curva de aprendizaje, cirujano entrenado vs bajo supervisión, además

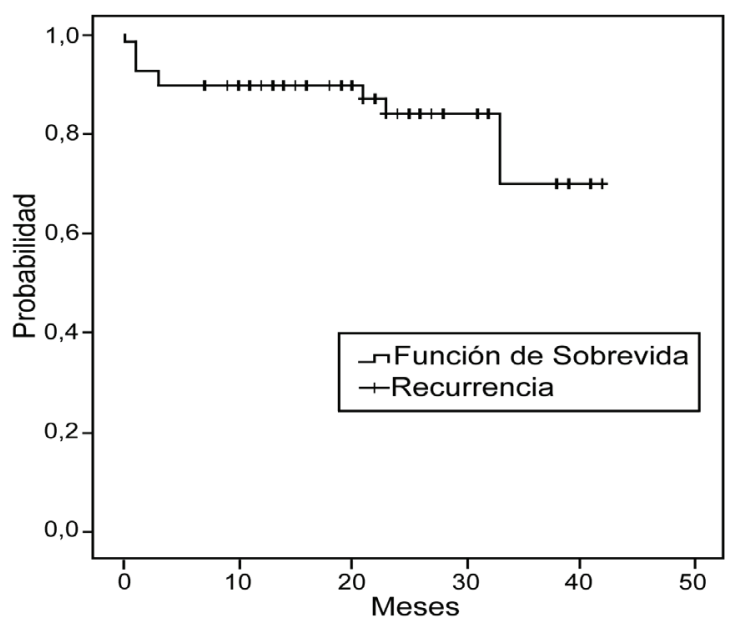

Figura 3. Curvas de sobrevida libre de recurrencia a 30 meses de seguimiento, en mujeres con colpoperineocleisis de Labhardt. 
Tabla IV

ANÁLISIS UNIVARIADO PARA RECIDIVA ANATÓMICA DE PACIENTES SOMETIDAS A COLPOPERINEOCLEISIS DE LABHARDT

\begin{tabular}{|c|c|c|c|}
\hline Variable & No recidiva & Recidiva & Valor $\mathrm{p}$ \\
\hline \multicolumn{4}{|l|}{ Antecedentes } \\
\hline Edad (años) & $71,8 \pm 5,7$ & $72,6 \pm 6,2$ & $0,7^{\mathrm{b}}$ \\
\hline Paridad total (Número de hijos) & $4,49 \pm 2,8$ & $4,2 \pm 2$ & $0,92^{c}$ \\
\hline RN mayor peso (gramos) & $3714 \pm 698$ & $3588 \pm 590$ & $0,6^{b}$ \\
\hline Parto instrumental & $12(20,6)$ & $2(20)$ & $1,0^{a}$ \\
\hline Antecedente de histerectomía & $8(13,7)$ & $2(20)$ & $0,634^{a}$ \\
\hline Antecedente cirugía por POP & $6(10,5)$ & $0(0)$ & $0,58^{a}$ \\
\hline Edad de menopausia (años) & $46,5 \pm 6,2$ & $46,8 \pm 3,7$ & $0,8^{b}$ \\
\hline $\mathrm{IMC} \mathrm{Kg/m2}$ & $26,9 \pm 5,2$ & $27,4 \pm 4,9$ & $0,8^{b}$ \\
\hline \multicolumn{4}{|l|}{ POPQ Preoperatorio } \\
\hline $\mathrm{Aa}$ & $1,4 \pm 1,7$ & $2 \pm 1,8$ & $0,27^{c}$ \\
\hline $\mathrm{Ba}$ & $3,5 \pm 2,2$ & $4,2 \pm 2,2$ & $0,79^{c}$ \\
\hline C & $1,4 \pm 4,2$ & $1,9 \pm 5,2$ & $0,92^{c}$ \\
\hline $\mathrm{gH}$ & $5,2 \pm 1,2$ & $5,5 \pm 1,6$ & $0,83^{c}$ \\
\hline $\mathrm{pB}$ & $2,9 \pm 0,8$ & $3,1 \pm 0,6$ & $0,33^{c}$ \\
\hline TVL & $7,9 \pm 1,2$ & $8,2 \pm 0,8$ & $0,6^{c}$ \\
\hline Ap & $-0,6 \pm-1,9$ & $-0,4 \pm 2,2$ & $0,93^{c}$ \\
\hline $\mathrm{Bp}$ & $0 \pm 2,7$ & $0,4 \pm 4$ & $0,97^{c}$ \\
\hline $\mathrm{D}$ & $-3 \pm 2,4$ & $-4,2 \pm 2,3$ & $0,3^{b}$ \\
\hline Prolapso pared anterior & $57(98,2)$ & $10(10)$ & $1,0^{a}$ \\
\hline Prolapso pared posterior & $48(82,7)$ & $9(90)$ & $1,0^{a}$ \\
\hline Prolapso apical & $49(84,4)$ & $9(90)$ & $0,5^{a}$ \\
\hline Prolapso multicompartimental & $55(94,8)$ & $10(100)$ & $1,0^{\mathrm{a}}$ \\
\hline \multicolumn{4}{|l|}{ Intraquirúrgico } \\
\hline Tiempo operatorio (min) & $54,8 \pm 19,3$ & $49,1 \pm 26,7$ & $0,4^{b}$ \\
\hline Pérdida sanguínea estimada (ml) & $74,6 \pm 72$ & $43 \pm 23,6$ & $0,076^{c}$ \\
\hline Qx staff & $43(74)$ & $7(70)$ & $0,71^{a}$ \\
\hline Qx concomitante & $7(12)$ & $1(10)$ & $1,0^{a}$ \\
\hline Curva aprendizaje & $8(13,7)$ & $3(30)$ & $0,34^{a}$ \\
\hline Respeto de técnica quirúrgica & $51(87,9)$ & $7(70)$ & $0,15^{a}$ \\
\hline \multicolumn{4}{|l|}{ Seguimiento } \\
\hline Meses de seguimiento & $20,9 \pm 8,3$ & $26,5 \pm 7,6$ & $0,05^{b^{*}}$ \\
\hline Flujo genital & $7(12)$ & $4(40)$ & $0,049^{a^{*}}$ \\
\hline
\end{tabular}

Qx: cirugía. * Diferencia significativa. ${ }^{a}$ Fisher exact. ${ }^{b}$ t-Student. ${ }^{c}$ Mann-Whitney U. Datos presentados como promedio $\pm \mathrm{DE}$ o número (porcentaje)

de la presencia de flujo vaginal en el seguimiento y el acatamiento de protocolo. Estas últimas 2 variables se incluyeron luego del análisis univariado dado que resultaron significativas. Las variables que mantuvieron significancia estadística fueron flujo vaginal $(p=0,026)$ con un OR: 6,3 (IC95\% 1,2-32) y el no acatamiento del protocolo establecido $(p=0,035)$ con un OR: 7,2 (IC95\% 1,1-45).

Al analizar los efectos sobre la sensación de satisfacción y calidad de vida, de las pacientes que no recidivaron, el $100 \%$ de las pacientes refirió me- joría. Ninguna de ellas refirió el empeoramiento de ésta poscirugía. En concordancia con ello, el 98,3\% de los casos se manifestó conforme o satisfecha de la cirugía realizada y el resultado obtenido.

\section{DISCUSIÓN}

La colpoperineocleisis de Labhardt es una antigua técnica reservada para pacientes sin actividad sexual. En nuestra serie, observamos que en pacientes bien seleccionadas, esta técnica ofrece muy 
buenos resultados en términos de reparación anatómica a un alto porcentaje de ellas $(85,3 \%)$ y asociado a una baja tasa de complicaciones. Es claro que estos resultados dependen del respeto a la técnica quirúrgica establecida, siendo cardinal la elección de las suturas correctas. De hecho en las pacientes donde se realizó la técnica quirúrgica sin modificaciones los resultados son mejores, reduciendo la recurrencia de 14,7 a 10,7\%.

Es importante enfatizar que los resultados de nuestro centro, muestran tasas de recidivas levemente mayores a las descritos en otras series que utilizan una técnica similar. En esas series, la tasa de recurrencia oscila entre 1 y $7 \%$ (16-18). Independiente de ello, nuestros resultados son alentadores comparado con los registros históricos y la experiencia personal que los cirujanos participantes han tenido con otras técnicas. A ello se agrega los resultados funcionales, que quedan reflejados en la sensación subjetiva de las pacientes. Ellas otorgan una alta tasa de satisfacción con la cirugía y en la mayoría de los casos hay mejoría en su calidad de vida.

Al comparar esta técnica con otras oclusivas, en particular con la colpocleisis de Le Fort, observamos tasas de recurrencia similares o levemente más alta a las publicadas por otros grupos, y que oscilan entre el 3 y $10 \%(11,14,20)$.

A la fecha no existen estudios aleatorizados que comparen las diferentes técnicas oclusivas. Creemos que todas las series, muchas de ellas retrospectivas, pueden contener un sesgo tanto de selección de las pacientes como también de seguimiento, todo lo cual influye en el registro de la recurrencia. La ventaja de nuestra serie, es que las pacientes incluidas corresponden a una población casi cautiva del sistema público de atención en salud. Ello queda refrendado porque el seguimiento de las pacientes es casi completo y 9 de las 10 recidivas, fueron operadas en el mismo centro realizando la misma técnica.

Es nuestra opinión que la colpoperineoclisis de Labhardt es una técnica potencialmente superior a la colpocleisis de Le Fort. Aunque los datos no avalan dicho planteamiento, nuestra opinión se fundamenta en un concepto anatómico. La operación de Le Fort se sustenta en la reparación del plano mucoso, mientras la operación de Labhardt busca reparar el defecto utilizando como mayor soporte una gran plicatura muscular, siendo secundaria la reparación mucosa. Esta hipótesis debe ser validada en el futuro mediante un estudio controlado aleatorizado que compare ambas técnicas.

Llama la atención que pese a ser esta una cirugía "definitiva", exista recurrencia sobre el 10\%, mayor al de otras series. Dentro de las posibles explicaciones se incluye la presencia de algunos factores predisponentes. Tal es el caso de la presencia de flujo vaginal, y por el cual se indicó tratamiento antibiótico. Este grupo de pacientes, en el análisis multivariado de COX, tuvieron 6,3 veces mayor riesgo de recurrir. Es difícil encontrar un fundamento biológico para esta situación. Especulamos dos alternativas: 1) la presencia de infección determina una respuesta inflamatorio defectuosa, con una mala cicatrización del plano muscular, lo cual predispone a la dehiscencia y posterior recidiva ó 2) la presencia de flujo es la expresión de una inflamación intensa, secundaria al desgarro del plano muscular, lo cual podría relacionarse a una técnica quirúrgica deficiente, y en la cual el proceso de cicatrización sería defectuoso en ausencia de infección. Ambas explicaciones podrían justificar el porcentaje más alto de recidiva observado en esta serie. Así también la mayor tasa de falla, puede ser explicada por el mayor tamaño muestral de nuestra serie, el seguimiento más prolongado con baja tasa de pérdida. Para confirmar o descartar el rol de la infección en la recidiva, resulta indispensable confirmar microbiológicamente la infección previo a indicar tratamiento antibiótico. Así también, es necesario comparar las tasas de recurrencia con el uso habitual de profilaxis (dosis única) vs el uso prolongado de antibiótico posoperatorio.

Otro punto relevante, al momento de analizar las recidivas, es la necesidad absoluta de ceñirse al protocolo. Ello incluye el no reemplazar suturas, incluyendo grosor y capacidad de reabsorción de éstas, así también el colocar correctamente las suturas en los planos profundos para obtener la correcta plicatura del elevador del ano. Este punto nos parece de extrema relevancia, pese a no haber alcanzado diferencias significativa en recurrencia al comparar la técnica adecuada con modificaciones de ella. El análisis multivariado de COX mostró que alejarse del protocolo establecido aumenta más de 7 veces el riesgo de recurrencia.

Un hecho destacable en nuestra serie, es el bajo porcentaje de 10 postoperatoria (5,9\% de los casos). Esta cifra es significativamente menor a lo reportado por otras series utilizando la técnica de Le Fort, y donde la IO alcanza hasta un $28 \%(11,21,22)$. Tan alta es la ocurrencia de IO con la técnica de Le Fort, que ha sido necesario agregar sling suburetrales a fin de alcanzar valores similares a los reportados por nuestra serie (13). El problema de adicionar cintas libres de tensión, es que podría asociarse a mayor tasa de complicaciones, además del considerable aumento en los costos en insumos. La diferencia en IO entre estas dos técnicas se explica porque en la 
cirugía de Labhardt no hay cambio alguno a nivel del cuello vesical, mientras que en la técnica de Le Fort si lo hay. Este es un punto que apoya la elección de la técnica de Labhardt en pacientes con POP sin actividad sexual, por sobre la colpocleisis de Le Fort.

Otro punto a destacar es una corta curva de aprendizaje, lo que se demuestra en no haber podido encontrar diferencias en tasa de recurrencia entre cirujanos expertos y cirujanos en curva de aprendizaje (bajo supervisión). Esta característica cobra relevancia en nuestro país, dado el escaso acceso a cirugías de alta complejidad, las que en muchas ocasiones tienen largas curvas de aprendizaje, como por ejemplo la colposacropexia, tanto abdominal como laparoscópica. Es importante señalar que, la no diferencia entre los primeros 5 y los posteriores procedimientos realizados por el mismo cirujano, podrían explicarse también por la baja incidencia del evento recurrencia como también por la definición de "curva de aprendizaje", lo que podría ocultar diferencias reales. Esto podría evaluarse de mejor manera al prolongar el seguimiento y utilizar como curva de aprendizaje una mayor cantidad de procedimientos.

Las limitaciones de nuestro trabajo están principalmente basadas en un diseño retrospectivo no comparativo. Esta situación impide sacar conclusiones que cambien conductas, sino solamente plantear nuevos estudios de mejor diseño que permitan comparar las distintas técnicas. Otra de la limitantes es que las mediciones de resultado subjetivos fue realizada con encuestas no validadas en población chilena.

Las principales fortalezas de nuestro trabajo están en el tamaño de la muestra y su seguimiento. Esta corresponde a la serie más extensa reportada en los últimos 40 años, superando en más del doble el número de pacientes de la serie que le sigue en tamaño (16). Nuestro seguimiento promedio es casi de dos años, y la tasa de pérdida es menor al $5 \%$ de la muestra.

\section{CONCLUSIÓN}

La colperineocleisis de Labhardt es una técnica segura, con bajo porcentaje de complicaciones y una tasa de recurrencia aceptable, comparable a la técnica de Le Fort. Nuestros resultados la apoyan como alternativa de manejo quirúrgico en pacientes bien seleccionadas, dado su alta tasa satisfacción quirúrgica, la menor tasa de 10 posoperatoria y su impacto positivo en la calidad de vida. Es necesario no modificar la técnica quirúrgica, lo que se asocia a mejores resultados anatómicos. Se requiere de estudios aleatorizados que comparen las distintas técnicas oclusivas, a fin de confirmar nuestra impresión de que esta técnica debiese ser de elección en pacientes con POP sin actividad sexual.

\section{REFERENCIAS}

1. Carey MP, Dweyer PL.Genital prolapse: vaginal versus abdominal route of repair. Curr Opin Obstet Gynecol 2001;13:499-505.

2. Digesu GA, Khullar V, Dudley C, Robinson L, Salvatore S. P-QOL: a validated questionnaire to assess the symptoms and quality of life of women with urogenital prolapse. Int Urogynecol J 2005;16:176-81.

3. Luber KM, Boero S, Choe JY. The demographics of pelvis floor disorders: current observations and future projections. Am J Obstet Gynecol 2001;184:1496-501.

4. Brown JS, Waetjen LE, Subak LL, Thom DH, Van den Eeden S, Vittinghoff E. Pelvic organ prolapse surgery in the United States. Am J Obstet Gynecol 1997;186:712-6.

5. Olsen A, Smith V, Bergstrom J. Epidemiology of surgically managed pelvic organ prolapse and urinary incontinence. Obstet Gynecol 1997;89:501-6.

6. Wu J, Hundley A, Fulton R, Myers E. Forecasting the prevalence of pelvic floor disorders in US women: 2010 to 2050. Obstet Gynecol 2009;114:1278-83.

7. Maher C, Feiner B, Baessler K, Glazener CM. Surgical management of pelvic organ prolapse in women. Cochrane Database Syst Rev 2010(04):CD004014.

8. FitzGerald M, Richter H, Siddique S, Thompson P, Zyczynski $\mathrm{H}$, Weber A, et al. Colpocleisis: a review. Int Urogynecol J 2006;17:261-71.

9. Abbasy S, Kenton K. Obliterative procedures for pelvic organ prolapse. Clin Obstet Gynecol 2010;53:86-98.

10. Reiffenstuhl G. Vaginal operations. En: Wilkins WA (ed). Surgical anatomy and technique. Baltimore;1996:161-80.

11. FitzGerald M, Brubaker L. Colpocleisis and urinary incontinence. Am J Obstet Gynecol 2003;189:1241-4.

12. Denehy T, Choe J, Gregori C, Breen J. Modified plication and compromised hysterectomy, posterior Le Fort partial colpocleisis with Kelly urethral posterior colpoperineoplasty in the medically elderly: A comparison with vaginal anterior colporrhaphy, and colpoperineoplasty. Am J Obstet Gynecol 1995;173:1697-702.

13. Smith A, Karp D, Lefevre R, Aguilar V, Davila W. LeFort colpocleisis and stress incontinence: weighing the risk of voiding dysfunction with sling placement. Int Urogynecol J 2011;22:1357-62.

14. Wheeler TL, Richter H, Burgio K, Redden D, Chen G, Goode P, Varner RE. Regret, satisfaction, and symptom improvement: Analysis of the impact of partial colpocleisis for the management of severe pelvic organ prolapse. Am J Obstet Gynecol 2005;193:2067-70.

15. Labhardt A. Kolpoperineokleisis subtotalis. Prolapsoperationen bei alte frauen. Zbl Gynäk. 1932;56:834-8.

16. Van Huisseling J. A modification of Labhardt's high perineoplasty for treatment of pelvic organ prolapse in the very old. Int Urogynecol J 2009;20:185-91.

17. von Massenbach W, Ohlenroth G. Resultate der subtotalen Kolpoperineokleisis nach Labhardt in der Mo- 
difikation nach H. Martius. Geburtshilfe Frauenheilkd. 1965;25(8):695-700.

18. Kilic G, Tunca J. Use of labhardt procedure to repair pelvic organ prolapse. Clin Exp Obst Gyn 2007;34 :91-2.

19. Bump RC, Mattiasson A, Bo K, Brubaker LP.The standadization of terminology of female pelvic organ prolapse and pelvic floor dysfunction. Am J Obstet Gynecol 1996;175:10-7.

20. Denehy T, Choe J, Gregori C, Breen J. Modified Le Fort partial colpocleisis with Kelly urethral plication and posterior colpoperineoplasty in the medically compromised elderly: A comparison with vaginal hys- terectomy, anterior colporrhaphy, and posterior colpoperineoplasty. Am J Obstet Gynecol 1995;173:1697702.

21. FitzGerald M, Richter H, Bradley C. Pelvic support, pelvic symptoms, and patient satisfaction after colpocleisis. Int Urogynecol J 2008;19:1603-9.

22. Nilsson C, Kuuva N, Falconer C, Rezapour M, Ulmsten U. Long-term results of the tension-free vaginal tape (TVT) procedure for surgical treatment of female stress urinary incontinence. Int Urogynecol J Pelvic Floor Dysfunct 2001;12(suppl 2):S5-S8. 